\title{
Behavioral Choice between Conflicting Alternatives Is Regulated by a Receptor Guanylyl Cyclase, GCY-28, and a Receptor Tyrosine Kinase, SCD-2, in AIA Interneurons of Caenorhabditis elegans
}

\author{
Yoichi Shinkai, ${ }^{1}$ Yuta Yamamoto, ${ }^{1}$ Manabi Fujiwara, ${ }^{1}$ Takashi Tabata, ${ }^{1}$ Takashi Murayama, ${ }^{1}$ Takaaki Hirotsu, ${ }^{1}$ \\ Daisuke D. Ikeda, ${ }^{2}$ Makoto Tsunozaki, ${ }^{3}$ Yuichi Iino, ${ }^{2}$ Cornelia I. Bargmann, ${ }^{3}$ Isao Katsura, ${ }^{4}$ and Takeshi Ishihara ${ }^{1,4}$ \\ ${ }^{1}$ Department of Biology, Graduate School of Sciences, Kyushu University, Fukuoka 812-8581, Japan, ${ }^{2}$ Department of Biophysics and Biochemistry, \\ Graduate School of Sciences, The University of Tokyo, Bunkyo-ku, Tokyo 113-0033, Japan, ${ }^{3}$ Howard Hughes Medical Institute, Laboratory of Neural \\ Circuits and Behavior, The Rockefeller University, New York, New York 10065, and ${ }^{4}$ Structural Biology Center, National Institute of Genetics, Mishima \\ 411-8540, Japan
}

Animals facing conflicting sensory cues make a behavioral choice between competing alternatives through integration of the sensory cues. Here, we performed a genetic screen to identify genes important for the sensory integration of two conflicting cues, the attractive odorant diacetyl and the aversive stimulus $\mathrm{Cu}^{2+}$, and found that the membrane-bound guanylyl cyclase GCY-28 and the receptor tyrosine kinase SCD-2 regulate the behavioral choice between these alternatives in Caenorhabditis elegans. The gcy-28 mutants and scd-2 mutants show an abnormal bias in the behavioral choice between the cues, although their responses to each individual cue are similar to those in wild-type animals. Mutants in a gene encoding a cyclic nucleotide gated ion channel, cng-1, also exhibit the defect in sensory integration. Molecular genetic analyses suggested that GCY-28 and SCD-2 regulate sensory integration in AIA interneurons, where the conflicting sensory cues may converge. Genetic ablation or hyperpolarization of AIA interneurons showed nearly the same phenotype as $g c y-28$ or $s c d-2$ mutants in the sensory integration, although this did not affect the sensory response to each individual cue. In $g c y-28$ or $s c d-2$ mutants, activation of AIA interneurons is sufficient to restore normal sensory integration. These results suggest that the activity of AIA interneurons regulates the behavioral choice between the alternatives. We propose that GCY-28 and SCD-2 regulate sensory integration by modulating the activity of AIA interneurons.

\section{Introduction}

Multimodal sensory cues should interact properly in our brain. These kinds of interactions are important to enhance the precision of perception (Meredith, 2002). On the other hand, the interaction is required in order for animals to choose behaviors when they receive conflicting sensory signals simultaneously (Meredith, 2002). For example, copulating behavior is suppressed by the perceived risk of predation and vice versa (Svenssona et al., 2007). The behavioral choice reflects the relative preference between the contradictory sensory cues (Ishihara et al., 2002; Certel et al., 2007; Svenssona et al., 2007; Kristan, 2008);

\footnotetext{
Received Sept. 5, 2010; revised Dec. 16, 2010; accepted Dec. 29, 2010.

This work was supported by a Grant-in-Aid for Scientific Research (T.I. and Y.I.), by Asahi Glass Foundation (T.I.), and by the Naito Foundation (T.I.). We thank A. Miyawaki, L. Garcia, M. Driscoll, and A. Fire for YC3.60, and unc$103(g f) C D N A$, mec-4(d) CDNA, and plasmids, respectively. We also thank S. Mitani for $g c y-28$ strains, D. Reiner for scd-2 strains and DNAs, the Caenorhabditis Genetic Center for strains, and Y. Kohara for $g c y-28$ cDNA.

Correspondence should be addressed to Takeshi Ishihara, Department of Biology, Graduate School of Sciences, Kyushu University, Hakozaki 6-10-1, Higashi-ku, Fukuoka 812-8581, Japan, e-mail:takeiscb@kyushu-u.org.

T. Murayama's present address: Okinawa Institute of Science and Technology, 0kinawa 904-0411, Japan.

M. Tsunozaki's present address: Department of Molecular and Cell Biology, University of California, Berkeley, CA 94720-3200.

DOI:10.1523/JNEUROSCI.4691-10.2011

Copyright $\odot 2011$ the authors $\quad 0270-6474 / 11 / 313007-09 \$ 15.00 / 0$
}

hence, the choice can be altered by an animal's status (Schall, 2001). Hunger increases the probability of food-seeking behavior, even at the cost of increased risk of becoming prey (Gillette et al., 2000). The trade-off between conflicting responses has been studied at the neural network level (Shaw and Kristan, 1997; Kristan, 2008). However, the dissection of behavioral choice through sensory integration at the molecular and cellular levels is often difficult in higher organisms because of their complex nervous systems.

Caenorhabditis elegans has a simple neural network composed of 302 neurons; the neuronal morphologies and their connections are well described (White et al., 1986), which enables us to assign functional and molecular properties to a specific neuron. For instance, sensory neurons called AWA, AWC, and ASE are involved in responses to an attractant (Bargmann et al., 1993); whereas, sensory neurons called ADL, ASH, and AWB function in the sensing and avoidance of repellents (Sambongi et al., 1999). Furthermore, calcium imaging studies have shown which neurons are activated by the presentation or removal of sensory stimuli (Suzuki et al., 2003, 2008; Kimura et al., 2004; Hilliard et al., 2005) and revealed information processing in local neural networks of C. elegans (Chalasani et al., 2007, 2010). 
In a previous study, we studied the response to two conflicting sensory signals, an attractive odorant diacetyl sensed by AWA neurons (Bargmann et al., 1993) and an aversive copper ion sensed by ASH and ADL neurons (Sambongi et al., 1999). We identified a secretory protein with a low-density lipoprotein (LDL) receptor motif, HEN-1, which regulates a sensory integration process non-cell autonomously in the mature neuronal circuit (Ishihara et al., 2002). Here, we describe additional regulators and neurons required for the sensory integration. We screened for mutants defective in the sensory integration and identified $g c y$-28, which encodes a receptor guanylyl cyclase. The receptor tyrosine kinase SCD-2 also regulates the sensory integration, probably as a receptor for HEN-1. Interestingly, both GCY-28 and SCD-2 regulate the sensory integration in a pair of interneurons called AIA, in which the conflicting sensory cues could converge. These studies not only reveal the mechanisms modulating sensory integration at the molecular and cellular levels, but also indicate that the activity of the integrative interneurons regulate the behavioral choice in the context of sensory integration.

\section{Materials and Methods}

Strains and culture. The strains used in this study were as follows: wild-type strain N2, gcy-28(qj4), gcy-28(tm2411), gcy-28(ky713), gcy28(ky887), gcy-28(tm3028), hen-1(tm501), glc-3(ok321), scd2(sa249), cng-1(jh111), cng-1(ok3292), CX9734 (gcy-28(ky713); kyEx2138 [podr-3:t01a4.1d::gfp]), CX10586 (gcy-28(ky713); kyEx2642 [pstr-2::t01a4.1d::gfp]), EG1470(oxEx229 [Mos1 Substrate, pmyo-2::gfp]), EG2762 (oxEx166 [HSP::MosTRANSPOSASE, lin$15(+)$, punc-122::gfp]).

All strains were cultured on NGM plates with E. coli strain OP-50 under standard conditions (Brenner, 1974). EG1470 and EG2762 were grown at $25^{\circ} \mathrm{C}$. The other strains were grown at $20^{\circ} \mathrm{C}$.

Screening for the mutants. Mos1-mediated insertional mutagenesis was performed as described previously (Boulin and Bessereau, 2007) with some modifications. Wild-type worms with both oxEx229 and oxEx166 were subjected to heat shock at $35^{\circ} \mathrm{C}$ for $75 \mathrm{~min}$. From the $\mathrm{F} 1$ progeny from eggs laid 12-16 h after the heat shock, $2520 \mathrm{~F} 1$ worms with oxEx229 were collected and allowed to lay F2 worms. The adult F2 worms were subjected to two rounds of interaction assays, using a 1:100 dilution of diacetyl and $30 \mathrm{mM} \mathrm{Cu}^{2+}$, to select mutants defective in the integration process. After the assays were finished, worms that did not cross the $\mathrm{Cu}^{2+}$ barrier were subjected to a chemotaxis assay to diacetyl (1:200 dilution) to exclude mutants defective in chemotaxis to diacetyl. In the chemotaxis assay, the worms that migrated to the spot of diacetyl were allowed to lay F3 worms, which were subjected to the two interaction assays and one chemotaxis assay according to the same protocol. After this set of behavioral assays was repeated a few times, animals defective in the interaction assay but not in chemotaxis were chosen as candidates for mutants defective in sensory integration. From these candidates, worms with Mos 1 insertion in the genome were selected for further analysis of their ability to perform chemotaxis and sensory integration. The Mos1 insertion of mutant qj4 was located in the fourth exon of $g c y-28$.

Behavioral assays. All data points are averages of more than six assays with $>50$ worms in each assay. The assays were performed on at least 3 different days. The asterisks indicate statistically significant differences $(p<0.01)$ relative to control. Statistical significance was determined by Student's $t$ test for the comparison between wild types and mutants and for the rescue experiments.

The interaction assay between chemotaxis to diacetyl and avoidance of $\mathrm{Cu}^{2+}$ ion was performed as described previously (Ishihara et al., 2002) with some modifications. Ten adult worms were put on a $6 \mathrm{~cm}$ NGM plate and allowed to lay eggs for $4 \mathrm{~h}$. After cultivation for $4 \mathrm{~d}$, adult worms were collected using S-basal buffer $(100 \mathrm{~mm} \mathrm{NaCl}, 50 \mathrm{~mm}$ potassium phosphate, $\mathrm{pH} 6,0.02 \%$ gelatin). The washed worms were placed on one side of the interaction assay plate, on which $\mathrm{a} \mathrm{Cu}^{2+}$ ion gradient had been formed at $25^{\circ} \mathrm{C}$ for $18-22 \mathrm{~h}$ by spreading $30 \mu \mathrm{l}$ of copper acetate solution on the midline of the assay plate. The assay was started by spotting $2 \mu \mathrm{l}$ of diluted diacetyl on the other side of the plate (see Fig. $1 A$ ). In this assay paradigm, to reach diacetyl, animals should cross the $\mathrm{Cu}^{2+}$ barrier. After $45 \mathrm{~min}$, the numbers of the worms on each side of the plate were counted. To evaluate the tendency to cross the $\mathrm{Cu}^{2+}$ barrier to reach diacetyl, the index was defined as the number of worms on the side of the diacetyl divided by the total number of the worms on the plate. In the assay, $1 \mu \mathrm{l}$ of $1 \mathrm{~m}$ sodium azide was placed on the attractant area. Unless otherwise indicated, 1:100 diluted diacetyl and $30 \mathrm{~mm}$ copper acetate were used in the interaction assay.

Chemotaxis toward the attractive odorants was based on Bargmann et al. (1993) except that the assay plate contained $50 \mathrm{~mm} \mathrm{NaCl}$. Chemotaxis index was calculated as described previously (Bargmann et al., 1993).

Avoidance of $\mathrm{Cu}^{2+}$ ion was investigated as described previously (Wicks et al., 2000) with some modifications. The worms washed four times with S-basal buffer and once with water were placed on the center of the quadrant assay plate (50 mM NaCl, $10 \mathrm{~mm}$ HEPES-Na, pH 7, $1 \mathrm{~mm}$ $\mathrm{MgSO}_{4}, 1 \mathrm{~mm} \mathrm{CaCl}, 2 \%$ agar), which was partitioned into four parts with or without $\mathrm{Cu}^{2+}$ ion. After $30 \mathrm{~min}$, the numbers of worms over the repellent area and the control area were counted. The index was calculated as described previously (Wicks et al., 2000). It was defined as the number of the worms over the repellent area minus the number of the worms over the control area, divided by the total number of the worms on the plate.

Heat shock experiments. $g c y$-28(qj4) mutants carrying phsp16.2::gcy-28 were cultivated at $20^{\circ} \mathrm{C}$ to young adulthood, and shifted to $33^{\circ} \mathrm{C}$ for $2 \mathrm{~h}$. The worms were cultured again at $20^{\circ} \mathrm{C}$ for $2 \mathrm{~h}$ and subjected to the interaction assay.

DNA constructs and germline transformation. gcy-28.c cDNA amplified by PCR was subcloned into the pPD49.26 vector. $g c y$-28.d cDNA, $p k c$ $1(g f) \mathrm{cDNA}$, and $g c y$ - 28 promoters were as described previously (Tsunozaki et al., 2008). $g l c-3$ cDNA was PCR amplified as described previously (Chalasani et al., 2007). For cell-specific RNA interference (RNAi), cng$1 . b$ cDNA amplified by PCR was inserted into Invitrogen Gateway destination vector in the sense orientation or in the antisense orientation. The $g c y$-28. $d$ promoter was used for the AIA-specific expression. For the expression analysis of $c n g-1$, the $4.6 \mathrm{~kb}$ upstream promoter region of $c n g-1$ gene amplified by PCR was inserted into pPD95.77 to generate $p c n g-1:: g f p$ construct. For the construction of genomic $s c d-2:: g f p$ fusion gene, green fluorescent protein (GFP) was fused just before the stop codon of the $s c d-2$ genomic fragment. The genomic fragment including $\sim 4 \mathrm{~kb}$ of upstream regulatory region was cloned into pMW118. The promoters were inserted into the upstream multicloning site of each plasmid. Transgenic strains were generated by microinjecting the DNA to be tested at a concentration of $10-75 \mathrm{ng} / \mu \mathrm{l}$ along with the $5 \mathrm{ng} / \mu \mathrm{l}$ cotransformation marker pmyo-3::gfp or plin-44::gfp and $0-85 \mathrm{ng} / \mu \mathrm{l}$ carrier DNA, as described by Mello and Fire (1995).

The fluorescence image of adult-stage hermaphrodites harboring pcng-1::gfp, genomic scd-2::gfp, or pgcy-28.d::gcy-28.d::gfp was obtained with a LSM 510 laser-scanning confocal microscope (Zeiss) or an Axioplan 2 microscope (Zeiss).

Calcium imaging. To generate an AWA imaging line, Cameleon YC 3.60 (Nagai et al., 2004) was expressed under the $o d r-10$ promoter (Sengupta et al., 1996). We confirmed that the expression of Cameleon in this line did not change the chemotactic behavior in response to diacetyl. Calcium imaging was performed using the microfluidic "olfactory" chip, as described previously (Chronis et al., 2007). Briefly, an animal was immobilized by trapping in a microchannel, and the nose of the animal was exposed to a flowing stream of diacetyl-containing or odor-free solutions. Fluorescent images of the calcium indicator were obtained using a Zeiss Axioplan 2 microscope equipped with $40 \times$ objective and a 3 CCD digital camera C7780 (Hamamatsu). All images were collected at 200 or $400 \mathrm{~ms}$ exposure time with no intervening interval, for 2-3 min total duration. Using the AquaCosmos software (version 2.6, Hamamatsu), the time stacks were analyzed for the yellow fluorescent protein (YFP) intensity/cyan fluorescent protein (CFP) intensity emission ratio in the AWA cell bodies. $R / R_{0}$ was calculated as the change in the YFP/CFP ratio 

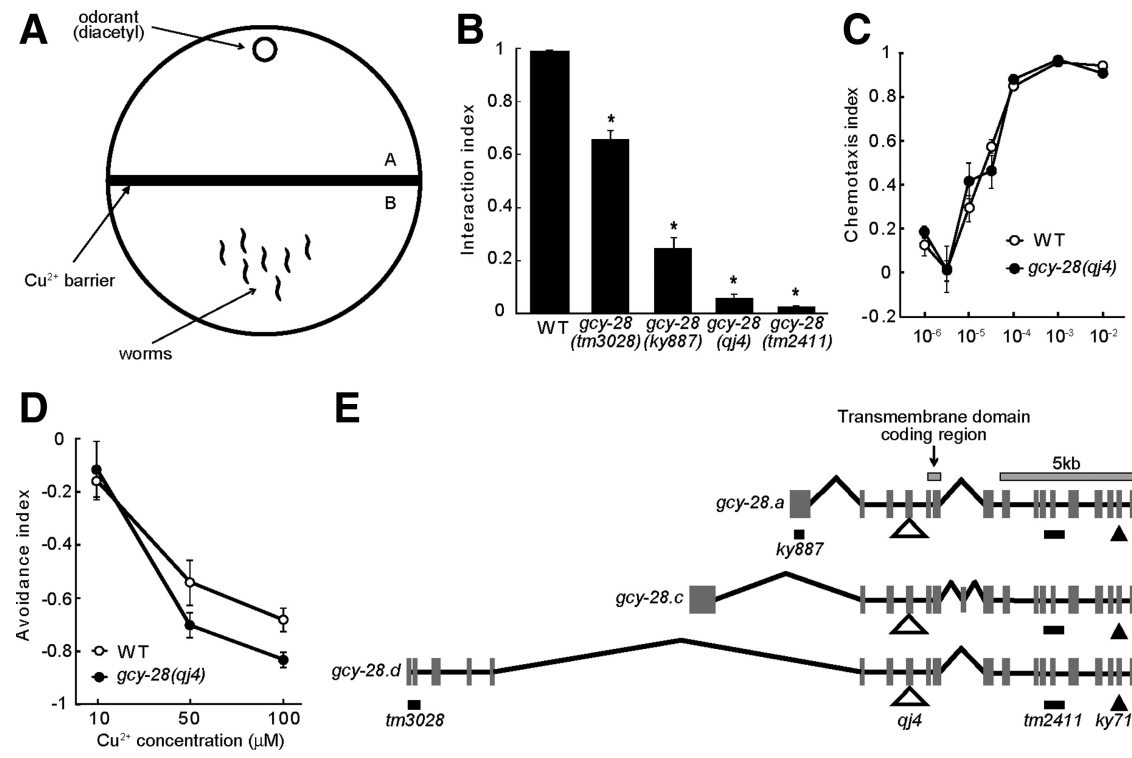

$\mathbf{E}$

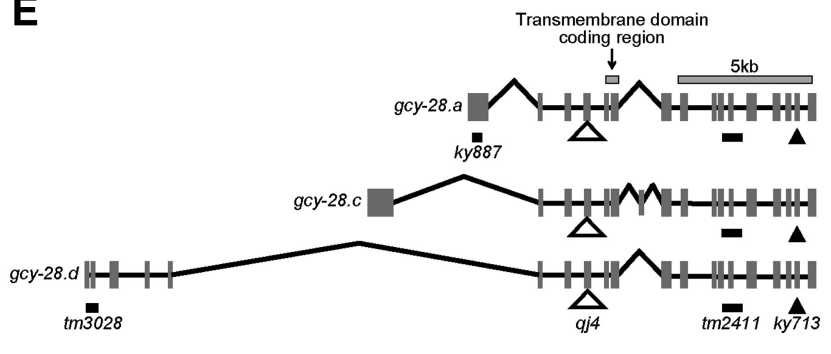

Figure 1. $g c y-28$ mutants show behavioral defects in the interaction of the two sensory signals. $\boldsymbol{A}$, Schematic diagram for an assay to test the interaction of two sensory signals, called the "interaction assay." Worms must cross aversive $\mathrm{Cu}^{2+}$ barrier spread on the midline of the assay plate to reach the spot containing the attractive odorant diacetyl. Forty-five minutes after the start of the assay, the numbers of worms on the original side (A) and on the odorant side (B) were scored. The index was calculated as $A /(A+B) . \boldsymbol{B}$, Phenotypes of $g c y-28$ mutants in the interaction assay. Error bars in $\boldsymbol{B}-\boldsymbol{D}$ indicate SEM. ${ }^{*} p<0.01$. C, Chemotaxis toward various concentrations of diacetyl of $g c y-28(q j 4)$ was analyzed by the conventional chemotaxis assay. $\mathbf{D}$, Avoidance of various concentrations of $\mathrm{Cu}^{2+}$ of $g c y-28(q j 4)$ was analyzed by the quadrant assay. In all points, the differences were not significant $(p>0.01)$. $\boldsymbol{E}$, Gene structure and mutations of $g c y-28$. Three alternative splice forms ( $g c y-28 . a, g c y-28 . c, g c y-28 . d)$ contain partly different $5^{\prime}$ exons encoding the extracellular domain. Black bars indicate deletions, and a filled triangle indicates a missense mutation. An open triangle indicates Mos 1 insertion. $k y 713$ missense mutation results in substitution of an amino acid in the conserved cyclase domain (Tsunozaki et al., 2008). The alleles $t m 3028, k y 887$, and $t m 2411$ are deletions predicted to cause a frameshift. Mos1 transposon insertion (qj4) is located in the fourth exon (I:4499749..4499750) and would result in premature termination of $\mathrm{GCY}-28$ protein at the extracellular domain. WT, Wild type.

$(R)$ relative to the mean basal ratio $\left(R_{0}\right)$ during $12 \mathrm{~s}$ preceding the first application of diacetyl.

\section{Results}

\section{GCY-28 regulates the integration of two sensory signals}

To investigate sensory integration, we used an interaction assay, in which an attractive odorant, diacetyl, and an aversive stimulus, $\mathrm{Cu}^{2+}$ ion, were used as conflicting sensory cues (Fig. 1A) (Ishihara et al., 2002). In this assay paradigm, animals must cross the $\mathrm{Cu}^{2+}$ barrier to reach the diacetyl; the percentage of animals that reach the diacetyl changes depending on the concentration of both $\mathrm{Cu}^{2+}$ and diacetyl. Therefore, by using this interaction assay, we can evaluate the sensory integration quantitatively (Ishihara et al., 2002). To identify genes regulating the sensory integration, we screened for mutants defective in the ability to cross the barrier in the interaction assay. Mutants were generated using the Mos1 transposon as a mutagen (Bessereau et al., 2001). The screen identified an allele, $q j 4$, which contains Mos1 insertion in the $g c y-28$ gene. $g c y$ - 28 mutants crossed the copper ion barrier in the interaction assay at much lower frequency than the wild type (Fig. $1 B$ ), although it appeared healthy. This phenotype could be explained by defects in the sensory integration, weaker response to diacetyl, or enhanced response to $\mathrm{Cu}^{2+}$. To distinguish among these possibilities, we analyzed the sensitivity of $g c y$-28( $q j 4)$ animals to diacetyl and to $\mathrm{Cu}^{2+}$, respectively. Since $g c y-28(q j 4)$ animals are devoid of three primal alternative splicing isoforms $(a, c$, and $d)($ Fig. $1 E)$ and show a stronger phenotype than other $g c y$ - 28 mutants, the role of all of the isoforms in the sensation of diacetyl and $\mathrm{Cu}^{2+}$ could be examined.
First, we analyzed chemotaxis in response to various concentrations of diacetyl and found that the response of $g c y-28(q j 4)$ animals to diacetyl was very similar to that of wild-type animals (Fig. 1C). Next, we examined the responses to $\mathrm{Cu}^{2+}$ in wild-type animals and $g c y$-28(qj4) and found that the avoidance response to $\mathrm{Cu}^{2+}$ in $g c y-28$ (qj4) animals was nearly the same as that in wildtype animals (Fig. 1D). These results suggested that $g c y$-28(qj4) animals have a defect in the integration of the two sensory signals.

\section{GCY-28 acts in the mature}

\section{AIA interneurons}

gcy-28 encodes a receptor-type guanylyl cyclase with an extracellular ligand-binding domain, a transmembrane domain, a protein kinase-like domain, and a guanylyl cyclase domain. Previous studies showed that GCY-28 regulates odor preferences in AWC sensory neurons (Tsunozaki et al., 2008). In addition, these studies reported that three splice isoforms $(a, c$, and $d)$, which share the intracellular domain but have distinct extracellular domains (Fig. 1E), are expressed in various tissues including the nervous system. Ortiz et al. (2006) analyzed the expression of $g c y$-28.c using a $g f p$ fusion gene and found that $g c y$-28.c is broadly expressed in the nervous system. We analyzed the expression of $g c y$-28.c and $g c y$-28.d using $g f p$ fusion genes. Although the $p g c y-28 . c:: g c y-28 . c:: g f p$ fusion gene was expressed in many neurons, $p g c y$-28.d::gcy-28.d::gfp fusion gene was expressed specifically in a pair of interneurons, AIA (Fig. $2 A$ ).

To examine whether GCY-28 in AWC sensory neurons regulates not only odor preference but also the behavioral choice, we analyzed the behavior of the $g c y-28$ mutant expressing GCY-28.d in AWC in the interaction assay, because the expression of GCY28.d in AWC can completely rescue the phenotype in the odor preference (Tsunozaki et al., 2008). gcy-28 animals carrying podr-3::gcy-28.d or pstr-2::gcy-28.d, both of which express GCY28.d in AWC ${ }^{\mathrm{ON}+\mathrm{OFF}}$ or AWC ${ }^{\mathrm{ON}}$, respectively, showed similar phenotypes as $g c y$ - 28 animals in the interaction assay, indicating that the odor preference in AWC does not affect the sensory integration between diacetyl and $\mathrm{Cu}^{2+}$ (Fig. $2 \mathrm{~B}$ ).

To identify neurons in which GCY-28 regulates sensory integration, we analyzed the sensory integration phenotype of $g c y-28$ animals expressing GCY-28 in various subsets of neurons by using the interaction assay. A $g c y-28 . c$ cDNA expressed by $\mathrm{H} 20$ pan-neuronal promoter rescued the defect of $g c y$-28(qj4) in the interaction assay (Fig. 2C), suggesting that $g c y-28$ regulates the sensory integration in the nervous system. Moreover, the expression of $g c y$-28.c cDNA in adults under the control of a heat shock promoter rescued the defect of $g c y-28(q j 4)$, indicating that the function of $g c y-28$ at adulthood is sufficient for normal sensory integration (Fig. 2D). To further investigate where $g c y$ - 28 acts in the nervous system, we analyzed the phenotypes of $g c y-28$ animals expressing $g c y$-28.c cDNA in various sets of neurons. These analyses showed that the defect in sensory integration could not be restored by the expression of $g c y-28 . c \mathrm{cDNA}$ in sensory neurons for diacetyl (AWA) or for $\mathrm{Cu}^{2+}$ ion (ASH, ADL) (Fig. 
A

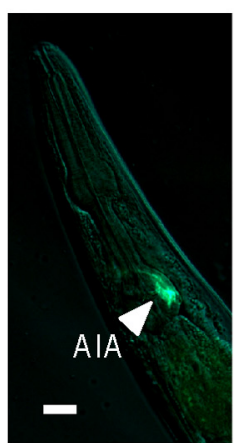

B

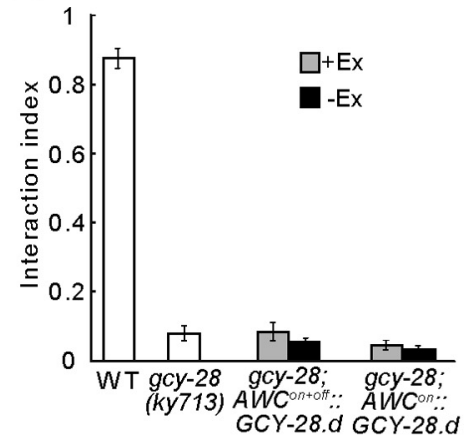

C

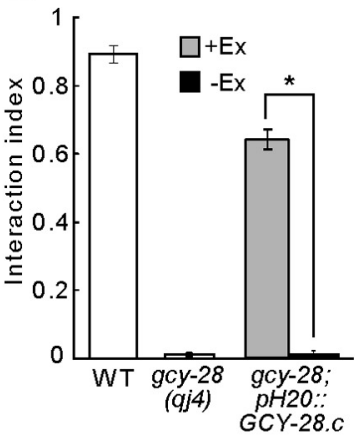

D

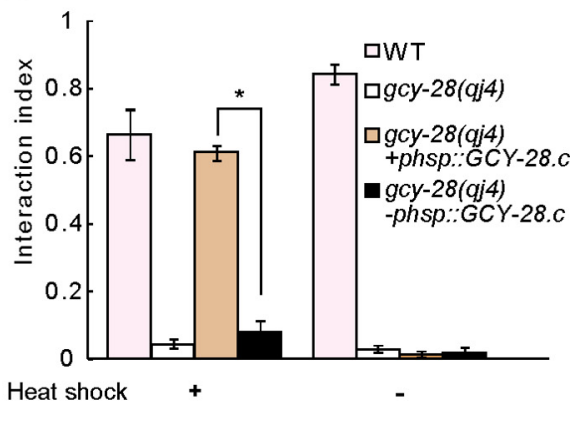

E

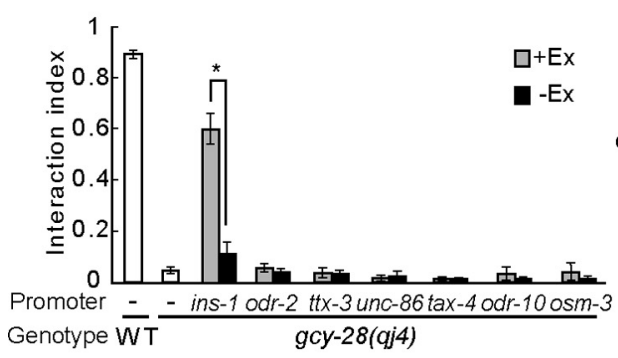

$F$

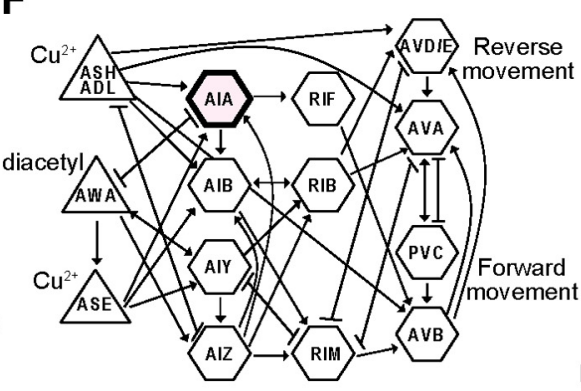

G

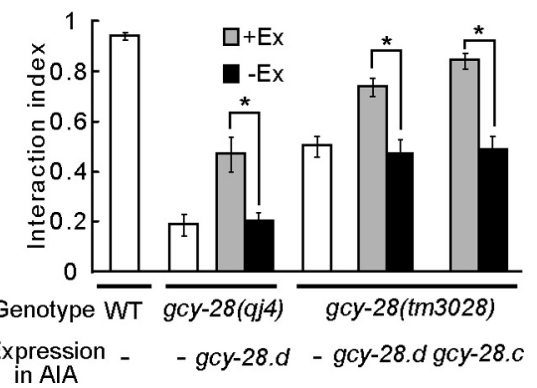

Figure 2. GCY-28 in the AlA interneurons is important for the interaction of two signals. $A$, The expression of the $g c y-28 . d:: g f p$ fusion gene under control of the $g c y-28 . d$ promoter at the adult stage. The site of expression is indicated by an arrowhead. Scale bar, $10 \mu \mathrm{m} . \boldsymbol{B}$, The phenotypes of $g c y-28$ mutant expressing $g c y-28 . d$ CDNA in AWC ${ }^{\text {ON }+ \text { OFF }}$ neurons or AWC ${ }^{\text {ON }}$ were analyzed by the interaction assay. Worms carrying the transgene ( $+E x$, gray columns) and worms not carrying the transgene ( $-E x$, black columns) were reared on the same culture plate and subjected to the assay on the same assay plate to compare the indices. White columns indicate nontransgenic worms reared on separate culture plates and subjected to the assay on separate assay plates. $\mathbf{C}-\boldsymbol{E}$, The phenotypes of $g c y-28$ mutants expressing $g c y-28$. c cDNA by various promoters were analyzed by the interaction assay. The expression of $H 20$ promoter: pan-neuron. The expression of ins- 1 promoter: neurons including AIA. The expression of unc-86 promoter: several neurons including AIZ. The expression of ttx-3 promoter: AIY. The expression of odr-2 promoter: neurons including AIB. The expression of tax-4, odr-10, or osm-3 promoter: sensory neurons including ADL, ASE, ASH, AWA, and AWC. gcy-28(qj4) animals carrying phsp::gcy-28.c were heat shocked at young adult stage. $\boldsymbol{F}$, The schematic diagram for the putative neural network for the interaction of two sensory signals, extracted from the complete circuit (White et al., 1986). Sensory neurons are shown as triangles and interneurons as hexagons. Arrows indicate chemical synapses, and lines with T-shaped ends indicate gap junctions. The AIA neurons expressing GCY-28.d are emphasized. G, $g c y-28$ mutants expressing $g c y-28$ CDNA in AIA interneurons by $g c y-28$. d promoter were analyzed in the interaction assay. Open bars show nontransgenic animals for comparison. $\boldsymbol{B}-\boldsymbol{E}, \boldsymbol{G},+$ Ex and - Ex worms were compared. Error bars in $\boldsymbol{B}-\boldsymbol{E}$ and $\boldsymbol{G}$ indicate SEM. ${ }^{*} p<0.01$. WT, Wild type.

$2 E, F)$. These results also supported the idea that $g c y$ - 28 mutants show a defect in the integration of the two sensory signals, rather than in the perception of those signals individually. On the other hand, $g c y-28$ animals that expressed $g c y$-28.c cDNA under control of ins- 1 promoter, which results in expression in multiple neurons including AIA interneurons (Tomioka et al., 2006), showed almost the same phenotype as wild-type animals in the interaction assay (Fig. $2 E$ ). These results suggested that specific neurons are involved in the sensory integration.

As GCY-28.d is mainly expressed in AIA interneurons, we analyzed the phenotypes of $g c y$-28(tm3028), in which $g c y$-28.dspecific exons are deleted and the deletion would lead to null mutation in $g c y$-28.d, in the interaction assay. We found that $g c y$-28(tm3028) showed the defect in the sensory integration (Fig. $1 B)$. Although the defect in $g c y-28(\operatorname{tm} 3028)$ was weaker than in the other $g c y$ - 28 mutants, these results raised a possibility that GCY-28.d in AIA interneurons is involved in the regulation of sensory integration. To examine this possibility, we investigated the behavior of $g c y$-28(tm3028) mutants that express $g c y-28$ cDNA in AIA. The expression of $g c y-28 . d$ cDNA in AIA can partially rescue the defect of $g c y-28(q j 4)$ mutants devoid of all GCY-28 isoforms, and significantly rescue that of $g c y-28(\mathrm{tm} 3028)$ mutants devoid of the GCY-28.d isoform (Fig. 2G). Moreover, the expression of $g c y$-28.c cDNA in AIA also rescued the defect of $g c y-28(t m 3028)$. These results suggested that GCY-28 in AIA interneurons is important for sensory integration.
CNG-1, a cGMP-gated ion channel, regulates the sensory integration

To further investigate the molecular mechanisms of the sensory integration in AIA interneurons, we used the interaction assay to analyze the phenotype of various mutants that are defective in the cGMP signaling pathway. These experiments were motivated by the finding that the mutation ky713 in the guanylyl cyclase domain of GCY-28 caused a defect in the sensory integration (Fig. $2 B$ ). Among those mutants, $c n g-1$, a mutant defective in a cGMPgated ion channel, showed a defect in sensory integration. Like gcy-28 mutants, cng- 1 animals showed a weaker tendency to cross the copper ion barrier than wild-type animals in the interaction assay (Fig. 3A), whereas their individual responses to diacetyl and $\mathrm{Cu}^{2+}$ are similar to that of wild-type animals (Fig. $3 B, C$ ). To determine the regulatory pathway of the sensory integration, the phenotype of $g c y$-28; $c n g-1$ double-null mutant animals was analyzed. For the generation of the double mutant, $g c y$-28(tm3028) allele was used to investigate genetic interaction between $g c y$ - 28 in AIA and $c n g-1$, because $c n g-1$ may function in AIA like GCY28.d (see next paragraph). $g c y$-28; $c n g-1$ double-mutant animals showed nearly the same phenotype as $c n g-1$ single-mutant animals (Fig. 3D). Therefore, $g c y-28$ and $c n g-1$ may operate in the same genetic pathway to regulate sensory integration.

A cng-1::gfp fusion gene is expressed in subsets of head neurons (Cho et al., 2005). To further characterize the expression pattern of CNG-1, we examined the expression pattern of the 
A
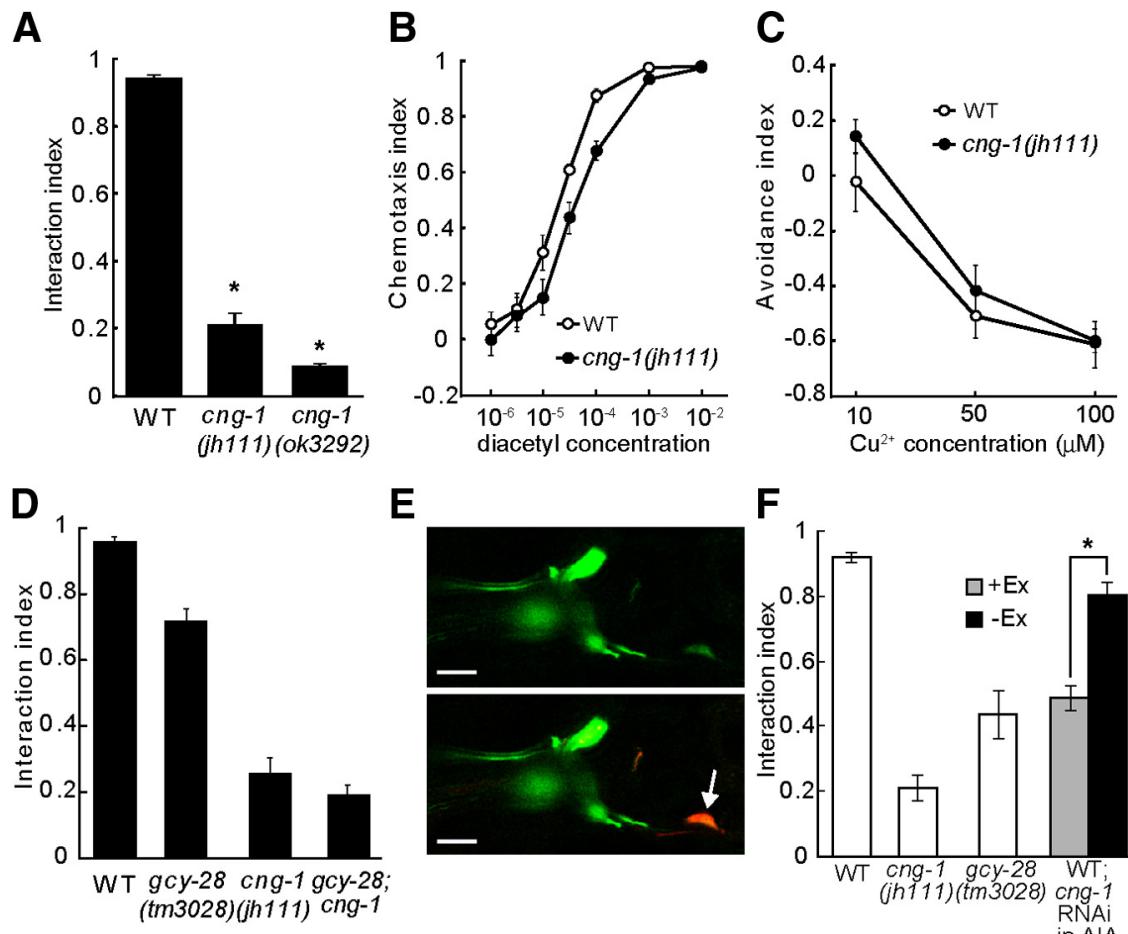

E
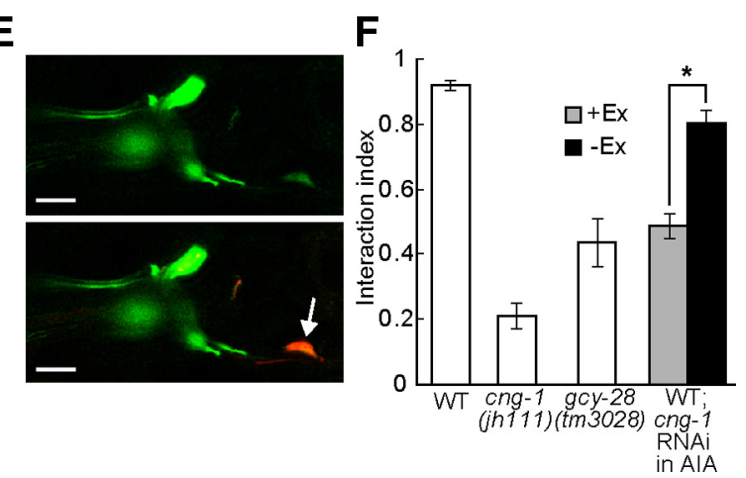

Figure 3. $g c y$-28 and cng-1 regulate the interaction of two sensory signals in the same genetic pathway. $A$, Phenotypes of $\mathrm{cng}$ - 1 mutants in the interaction assay. Error bars in $\boldsymbol{A}-\boldsymbol{D}$ and $\boldsymbol{F}$ indicate SEM. ${ }^{*} p<0.01$. $\boldsymbol{B}$, Chemotaxis toward various concentrations of diacetyl of $\mathrm{Cng}-1$ was analyzed by the conventional chemotaxis assay. $\boldsymbol{C}$, Avoidance of various concentrations of $\mathrm{Cu}^{2+}$ of $\mathrm{Cng}-1$ was analyzed by the quadrant assay. $\boldsymbol{D}$, The genetic interaction between $\mathrm{cng}-1$ and $g c y-28$ in the interaction assay (15 mu copper acetate was used as $\mathrm{Cu}^{2+}$ barrier). $\boldsymbol{E}$, Confocal views of wild-type animals expressing GFP under the control of the cng-1 promoter at the adult stage. The expression of GFP was colocalized with monomeric red fluorescent protein expressed in AIA by ins-1 promoter (bottom, arrow). Scale bars, $10 \mu \mathrm{m}$. F, RNAi knockdown of $c n g-1$ in AIA interneurons of wild-type animals; their behavior was analyzed in the interaction assay. Worms carrying the transgene ( $+\mathrm{Ex}$ ) and worms not carrying the transgene ( $-\mathrm{Ex}$ ) were compared. WT, Wild type.

transcriptional reporter fusion. Expression was observed in subsets of head neurons including AIA interneurons (Fig. 3E), suggesting the role of CNG-1 in sensory integration within AIA interneurons. To determine whether $c n g-1$ regulates sensory integration in AIA interneurons, we knocked down $c n g-1$ in AIA interneurons by using cell-specific RNAi (Esposito et al., 2007). Transgenic worms expressing sense and antisense RNAs corresponding to $c n g-1$ only in AIA crossed the $\mathrm{Cu}^{2+}$ barrier at lower frequency than wild type (Fig. $3 F$ ). The defect of the transgenic worms was comparable to that of $g c y$-28(tm3028) mutants, in which $g c y$-28. $d$-specific exons are deleted. These results suggested that CNG-1 in AIA interneurons is important for sensory integration like GCY-28.

\section{HEN-1/SCD-2 pathway regulates the integration of two sensory signals in AIA interneurons in parallel to GCY-28} We previously identified HEN-1 as a regulator of sensory integration by using the interaction assay (Ishihara et al., 2002). hen-1 encodes a secretory protein with an LDLa motif similar to that of Jeb protein in Drosophila. Jeb was reported to regulate development of visceral mesodermal cells via the tyrosine kinase receptor DAlk (Lee et al., 2003). The worm genome contains only one gene for a tyrosine kinase receptor similar to DAlk, $s c d-2$ (Reiner et al., 2008). Furthermore, hen-1 and scd-2 mutants showed nearly the same phenotype in dauer formation, suggesting that SCD-2 is a receptor for HEN-1 (Reiner et al., 2008). Therefore, we explored the function of SCD-2 in sensory integration. We found that scd-2(sa249)-null mutants tended not to cross the copper ion barrier in the interaction assay, just as with hen-1(tm501)-null mutants (Fig. $4 A)$. The responses of $s c d-2$ animals to diacetyl or copper ions individually were indistinguishable from those of wild-type animals (Fig. $4 B, C$ ). Next, we analyzed the phenotype of scd-2;hen-1 double-mutant animals in the interaction assay and found that scd-2;hen-1 double-mutant animals showed a phenotype similar to each of the single mutants, suggesting that hen-1 and $s c d-2$ act in the same genetic pathway (Fig. $4 A$ ), as they do to regulate dauer formation (Reiner et al., 2008).

To elucidate the expression pattern of SCD-2, we analyzed the expression pattern of the functional $g f p$ fusion gene and found that SCD-2 was expressed in many neurons including AIA interneurons (Fig. 4D). To determine whether the expression of SCD-2 in AIA is sufficient for the proper sensory integration, we analyzed scd-2 mutant animals expressing $s c d-2$ cDNA under the control of the $g c y-28 . d$ promoter and found that the expression of SCD-2 in AIA significantly restored the phenotype of $s c d$-2 in the interaction assay (Fig. 4E). On the other hand, expression of this gene in other neurons was unable to restore the phenotype (data not shown). These results suggest that SCD-2 in AIA interneurons is important for the regulation of sensory integration behavior.

As both $g c y$-28 and $s c d-2$ function in AIA interneurons, these molecules could act in the same genetic pathway. To examine this possibility, we constructed the $g c y$-28(tm3028);scd2(sa249) double-null mutant and analyzed its behavior in the interaction assay. $g c y$-28; $s c d-2$ double-mutant animals showed a more severe defect than single-mutant animals in the interaction assay, suggesting that GCY-28 and SCD-2 function in distinct pathways (Fig. $4 F$ ).

\section{AIA interneurons are necessary and sufficient for sensory integration}

To determine the role of the AIA interneurons in the sensory integration, we analyzed the behavior of worms lacking AIA interneurons in the interaction assay. The loss of AIA interneurons would cause a behavioral change in the interaction assay. To disrupt AIA interneurons, we used a hyperactive form of MEC-4, called MEC-4(d). MEC-4 is a DEG/ENaC sodium channel, and the expression of MEC-4(d) in neurons causes neuronal toxicity in C. elegans (Harbinder et al., 1997). Most worms expressing MEC-4(d) exhibited degenerating AIA neurons (25/32) and did not cross $\mathrm{Cu}^{2+}$ barrier as much as wild-type animals in the interaction assay (Fig. 5A), whereas those animals show normal responses to the each individual cue (Fig. $5 B, C$ ). These results suggested that AIA interneurons are required for the proper regulation of the sensory integration.

We next examined whether inactivation of AIA interneurons affects the sensory integration behavior. To this end, we used the expression of UNC-103 [gain of function (gf)], a constitutively active form of the ERG-like potassium channel UNC-103, which could hyperpolarize the neurons and reduce their ability to transmit sig- 
nals (Reiner et al., 2006). Inactivation of the AIA interneurons by expressing UNC103 (gf) in AIA caused a defect in the sensory integration; animals expressing UNC103(gf) in AIA neurons showed a weaker tendency to cross the $\mathrm{Cu}^{2+}$ barrier like gcy-28 animals (Fig. 5A), although the responses to each individual cue in these animals are indistinguishable from those of wild-type animals (Fig. $5 B, C$ ). These results suggested that the activity of AIA is important for proper regulation of the sensory integration and does not affect the sensory responses.

We then asked whether the activity of the AIA interneurons is important for the regulation of the sensory integration. The expression of constitutively active protein kinase C [PKC-1(gf)], which may positively regulate synaptic transmission and neuropeptide release (Sieburth et al., 2005, 2007), can activate neurons in C. elegans (Okochi et al., 2005; Macosko et al., 2009). We found that the expression of PKC-1(gf) in AIA interneurons is sufficient to restore the phenotype of $g c y$-28 animals and $c n g-1$ animals in the interaction assay (Fig. 5D). Furthermore, the expression of PKC1 (gf) can also restore the phenotype of scd-2 (Fig. 5E). These results suggested that activation of synaptic release by AIA interneurons can bypass regulatory defects in sensory integration. On the other hand, the expression of PKC-1(gf) did not affect the behavior of wild-type worms in the interaction assay (Fig. 5F). Therefore, the expression of PKC-1(gf) may activate functions of the AIA interneurons and, hence, can restore the phenotypes. This implicates the possibility that activation of the AIA interneurons induces animals to cross the $\mathrm{Cu}^{2+}$ barrier to reach diacetyl.

The activity of AIA interneurons might be regulated by the balance between two conflicting sensory cues, diacetyl and $\mathrm{Cu}^{2+}$

The signals for the two conflicting sensory cues used in the interaction assay could converge in AIA interneurons. AIA interneurons connect with the AWA sensory neurons, which are involved in the sensation of diacetyl (Bargmann et al., 1993), via gap junctions; in contrast, AIA interneurons receive chemical synapses from the ASH/ADL sensory neurons, which are involved in the sensation of the $\mathrm{Cu}^{2+}$ ion (Fig. 2 F) (White et al., 1986; Sambongi et al., 1999).

Previous studies indicated that the AWA neurons express the receptor for diacetyl, ODR-10 (Sengupta et al., 1996; Dwyer et al., 1998), but the response of the AWA sensory neurons remains unknown. To study this, we used the genetically encoded calcium indicator Cameleon YC3.60, which changes the ratio of YFP/CFP fluorescence upon calcium binding (Miyawaki et al., 1997). We found that intracellular calcium concentration in AWA neurons is transiently increased by stimulation with diacetyl (Fig. 6A). This result suggests that the AIA interneurons could be activated through gap junctions between AIA and AWA upon exposure to diacetyl.
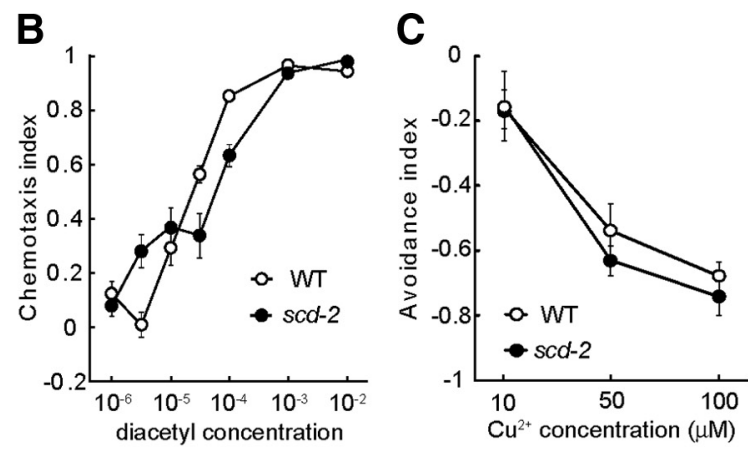

E
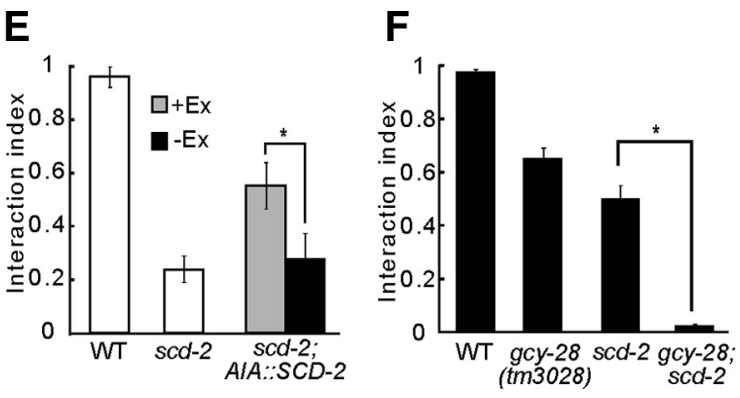

Figure 4. SCD-2 in AIA interneurons is important for the interaction of two sensory signals. $A$, The genetic interaction between 促 列 was analyzed by the interaction assay. Worms carrying the transgene $(+\mathrm{Ex})$ and worms not carrying the transgene

In ASH neurons, intracellular calcium ion increases as a function of stimulation by $\mathrm{Cu}^{2+}$ (Hilliard et al., 2005), but the synaptic property between the ASH neurons and the AIA interneurons has been unknown. To examine the property, as ASH sensory neurons are known to be glutamatergic, and the L-glutamate-gated chloride 3 (GLC-3) channel acts in the AIA interneurons (Chalasani et al., 2010), we analyzed the phenotype of the $g l c-3$ mutant. $g l c$-3 mutants crossed the barrier more frequently than wild-type animals (Fig. $6 B)$. Furthermore, animals expressing GLC-3 in AIA interneurons could rescue the phenotype of the $g l c-3$ mutant (Fig. 6B). These results suggested that GLC- 3 mediates the inhibitory synaptic inputs from the ASH sensory neurons to the AIA interneurons. In contrast, loss of $g l c$ - 3 function in the $g c y$ - 28 mutant did not cause the worms to cross more frequently than the $g c y-28$ mutant (Fig. $6 \mathrm{~B}$ ), suggesting that GCY-28 acts downstream of GLC-3 in the sensory integration. This result is consistent with the idea that GCY-28 regulates the neuronal activity of AIA interneurons. In conclusion, the AIA interneurons could regulate the neuronal activity and the synaptic output, depending on the balance between the signals from the AWA sensory neurons and those from the ASH sensory neurons (Fig. 6C).

\section{Discussion}

\section{Regulators of the sensory integration}

Sensory integration is a simple way of processing sensory information, in which two sensory inputs are processed into one output signal. Proper sensory integration is important for proper behavioral choices. To elucidate the mechanisms of sensory integration at the molecular and neuronal levels, we studied the behavior of C. elegans exposed to both an attractive odorant, diacetyl, and an aversive stimulus, $\mathrm{Cu}^{2+}$ ion. Molecular genetic analyses of the sensory integration revealed that two genetically distinct pathways modify the integra- 
A

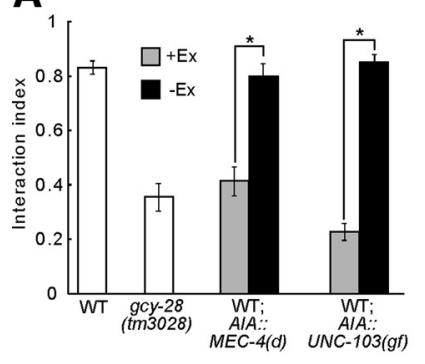

B

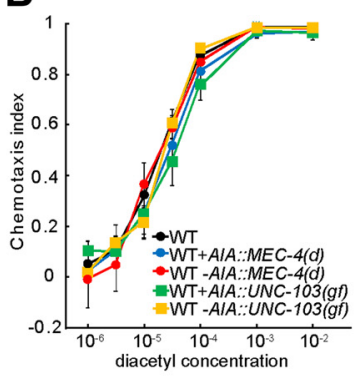

E

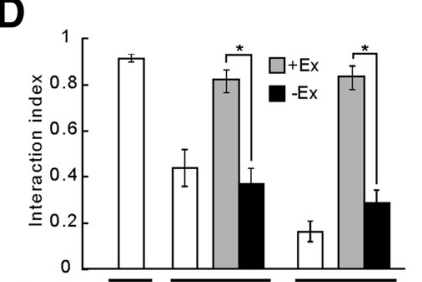

Genotype $\overline{W T} \overline{g c y-28(t m 3028)} \overline{c n g-1(j h 111)}$ Expression
of PKC-1(gf)

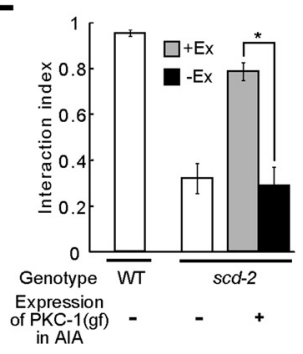

C

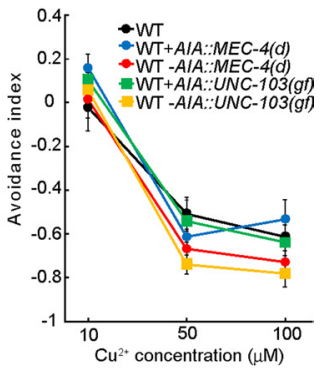

$\mathbf{F}$

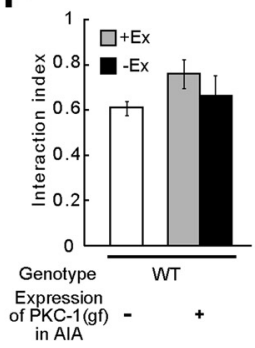

Figure 5. The AIA interneurons regulate sensory integration. $\boldsymbol{A}$, The functions of AIA interneurons were inhibited by the expression of MEC-4(d) or constitutively active UNC-103 in wild-type animals; their behavior was analyzed in the interaction assay. $\boldsymbol{B}$, Chemotaxis toward various concentrations of diacetyl of worms expressing MEC-4(d) or UNC-103 (gf) in AIA was analyzed by the conventional chemotaxis assay. $\boldsymbol{C}$, Avoidance of various concentrations of $\mathrm{Cu}^{2+}$ of worms expressing MEC-4(d) or UNC-103(gf) in AIA was analyzed by the quadrant assay. All points showed no significant differences from wild type $(p>0.01)$. $\boldsymbol{D}, \boldsymbol{E}$, The expression of constitutively active PKC- 1 in AIA interneurons could rescue the defects of $g c y$ - 28 mutants, scd- 2 mutants, and cng-1 mutants in the interaction assay. $\boldsymbol{F}$, The effect of the expression of constitutively active PKC- 1 in AIA interneurons on the phenotype of wild-type worms in the interaction assay ( $100 \mathrm{~mm}$ copper acetate was used as $\mathrm{Cu}^{2+}$ barrier). $\boldsymbol{A}-\boldsymbol{F}$, Worms carrying the transgene $(+\mathrm{Ex})$ and worms not carrying the transgene ( - Ex) were compared. Error bars in $\boldsymbol{A}-\boldsymbol{F}$ indicate SEM. ${ }^{*} p<0.01$. WT, Wild type.

A

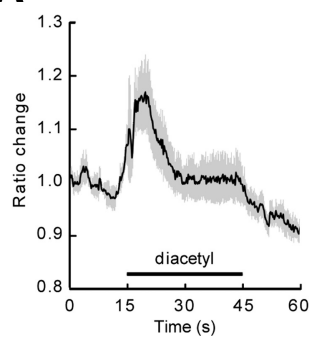

B

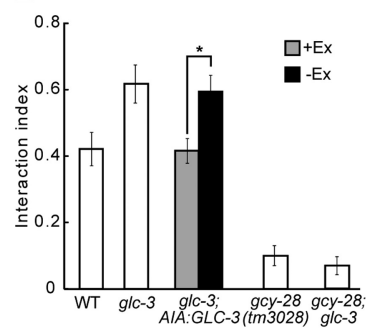

C

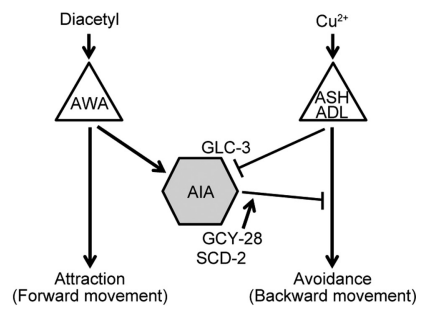

Figure 6. AIA interneurons receive excitatory and inhibitory inputs from distinct sensory neurons. $A$, Calcium responses of AWA sensory neurons to diacetyl measured by the $\mathrm{Ca}^{2+}$-sensitive fluorescent indicator Cameleon in wild-type animals. The black line indicates the average trace, and the shaded areas around the trace represent SEM ( $n=3$ animals). The blackbar indicates the presence of diacetyl. $\boldsymbol{B}$, The effect of mutations in glutamate-gated chloride channel GLC -3 on the behavior of wild-type animals and $g c y$ - 28 mutants in the interaction assay (100 mm copper acetate was used as $\mathrm{Cu}^{2+}$ barrier). Worms carrying the transgene (+Ex) and worms not carrying the transgene ( - Ex) were compared. Error bars indicate SEM. ${ }^{*} p<0.01$. C, A neuronal circuit model for the interaction of two sensory signals. Sensory neurons are shown as triangles, and interneurons, as hexagons. Arrows indicate excitatory connections, and lines with T-shaped ends indicate inhibitory connections. The activity of AIA interneurons would be regulated by the balance between excitatory inputs from AWA through gap junction and inhibitory inputs from ADL/ASH through GLC-3. Activated AIA interneurons would increase the relative strength of the signals for diacetyl by inhibiting the signals for $\mathrm{Cu}^{2+}$. GCY-28 and SCD-2 may enhance the inhibitory synaptic outputs from AIA.

tion of the conflicting sensory cues, the GCY-28/CNG-1 pathway and HEN-1/SCD-2 pathway. We also found that GCY-28.d and SCD-2 function in a pair of interneurons, AIA, in which sensory signals from AWA sensory neurons (responding to diacetyl) and from ADL/ASH sensory neurons (responding to $\mathrm{Cu}^{2+}$ ion) converge. Our results suggested that the neuronal activity of AIA interneurons regulates sensory integration to generate the appropriate behavioral choice.

GCY-28 may modulate a neuronal function in the nervous system. Most of the 34 guanylyl cyclases identified in C. elegans are tween forming a dauer or an L3 larva.

expressed in sensory neurons, and are thought to function as chemoreceptors or downstream signaling molecules in the sensing of substances in the environment (Inada et al., 2006; Ortiz et al., 2006, 2009; Zimmer et al., 2009). However, GCY-28 functions as a sensory regulator but not as a sensory receptor in AWC sensory neurons (Tsunozaki et al., 2008). gcy-28 encodes the guanylyl cyclase that is most similar to natriuretic peptide receptor-A (NPR-A) and -B. In mammals, NPR-A and -B modulate neurotransmission as receptors for natriuretic peptides, which are synthesized and secreted depending on the context (Herring and $\mathrm{Pa}$ terson, 2009). We found that GCY-28 is expressed and functions in interneurons, where it may regulate sensory integration by binding an unknown ligand. Domain-swap experiments also suggest that the guanylyl cyclase of GCY-28 can be activated by endogenous peptides (Baude et al., 1997). While there are no obvious homologs of natriuretic peptides in the C. elegans genome, an unknown ligand secreted in specific contexts may enable worms to change their behavioral choices on the basis of previous experience and current status.

$s c d-2$ encodes the C. elegans homolog of an anaplastic lymphoma kinase (ALK). Mammalian ALK has been postulated to function in the nervous system because of its expression in the adult brain (Iwahara et al., 1997; Bilsland et al., 2008; Palmer et al., 2009). A putative ligand for ALK plays a role in synaptic plasticity (Amet et al., 2001; Pavlov et al., 2006). In C. elegans, the HEN-1/ SCD-2 pathway modulates dauer larva formation, which is induced by harsh conditions such as high temperature, food deprivation, and crowding (Reiner et al., 2008). Food supply reduces the frequency of dauer formation induced by high population density of worms (Golden and Riddle, 1984). The entry into dauer larva stage happens under conditions of a low ratio of food to population density, implying that worms integrate environmental stimuli in the nervous system to choose their developmental fate. As the HEN-1/SCD-2 pathway regulates the sensory integration for the behavioral choice, it also might participate in the regulation of the developmental choice be-

GCY-28 and SCD-2 are expressed in many neurons. Our results suggested that GCY-28 and SCD-2 regulate integrative behavior by modulating the activity of AIA. Killing AIA interneurons caused a defect not only in integration but also in salt chemotaxis learning (Tomioka et al., 2006), suggesting that AIA interneurons are important for behavioral plasticity. However, it is likely that GCY-28 and SCD-2 also modulate the function of other interneurons that integrate sensory signals other than $\mathrm{Cu}^{2+}$ and diacetyl. Upon thermotaxis, opposing cryophilic and thermophilic driving signals are 
integrated in RIA interneurons (Mori et al., 2007). Mutations in gcy-28 or hen-1 encoding a ligand for SCD-2 individually cause a defect in food-associated thermotactic behavioral plasticity (Ishihara et al., 2002; Kodama et al., 2006; Tsunozaki et al., 2008), consistent with an important role for GCY-28 and SCD-2 in modulating neural circuits involved in integrative behaviors.

\section{AIA interneurons integrate multiple sensory cues to adjust behavioral choices}

Our results suggested that AIA interneurons are important for proper sensory integration. Because these interneurons receive synaptic inputs from multiple sensory neurons, the AIA interneurons can act as an integrator for such inputs. AWA sensory neurons, which are activated by diacetyl, make gap junctions with AIA interneurons (White et al., 1986); hence, AIA interneurons could be activated by diacetyl. On the other hand, ASH and ADL sensory neurons, which are activated by $\mathrm{Cu}^{2+}$ (Sambongi et al., 1999; Hilliard et al., 2005), may inhibit AIA interneurons through the glutamate-gated chloride channel GLC-3. We propose that, depending on the balance of these conflicting signals from AWA and ASH/ $\mathrm{ADL}$, the synaptic transmission from AIA is regulated by the HEN1/SCD-2 pathway and by GCY-28 pathway for the proper behavioral choice. In $g c y$-28 mutants, the activity of AIA neurons tends to be lower than that in wild-type animals, thereby decreasing synaptic transmission. Therefore, the mutants may behave in the interaction assay as if the worms sensed a higher ratio of $\mathrm{Cu}^{2+}$ to diacetyl than wild-type animals (Fig. 6C). In mammals, cyclic nucleotide-gated ion channels regulate the synaptic transmission and the excitability of olfactory receptor neurons (ORNs) (Murphy and Isaacson, 2003). By the expression of a constitutively active form of PKC-1, the activation of AIA rescued the defects of $g c y-28$ mutants and $c n g-1 \mathrm{mu}-$ tants in the sensory integration. This observation is consistent with the hypothesis that GCY-28 activates AIA interneurons to facilitate the synaptic transmission.

\section{The neural circuit for the sensory integration}

AIA interneurons, in which GCY-28/CNG-1 and HEN-1/SCD-2 regulate the sensory integration, are amphid interneurons. How do AIA interneurons regulate the sensory integration? In the interaction assay, the percentages of animals that reach the diacetyl change depend on the concentration of both $\mathrm{Cu}^{2+}$ and diacetyl (Ishihara et al., 2002), suggesting that the signals for the two conflicting cues suppress each other. Here, we demonstrated that $g c y-28$ and $s c d-2 \mathrm{mu}-$ tants showed normal responses to $\mathrm{Cu}^{2+}$ or diacetyl individually. Furthermore, the genetic ablation or inactivation of AIA interneurons of wild-type animals caused behavior similar to $g c y-28$ or $s c d-2$ mutants in the interaction assay, although this did not affect the sensory responses to each individual cue. Therefore, we propose that the AIA interneurons, where GCY-28 and SCD-2 modulate activity, are important for mutual inhibition of conflicting cues, but not for transduction of each sensory cue.

Our results suggested that the AIA interneurons are important to regulate the behavioral choice between crossing the $\mathrm{Cu}^{2+}$ barrier to reach diacetyl and not crossing the barrier (Fig. 5). Although AIA interneurons send synaptic outputs onto many neurons, the major synaptic target of AIA is AIB interneurons (White et al., 1986), which regulate reversal movement; removal of odors sensed by AWC neurons activates AIB interneurons and induces a reversal movement (Chalasani et al., 2007). On the other hand, the presentation of such odors causes activation of AIA interneurons and forward movement (Chalasani et al., 2010). Therefore, AIA and AIB exhibit opposite functions on behavior, suggesting that AIA sends inhibitory synapses onto AIB. Inhibitory connectivity from AIA to AIB is also predicted by the laser ablation studies (Wakabayashi et al., 2004). AIB interneurons receive synaptic inputs from ASH and ADL neurons that sense aversive chemicals (White et al., 1986), suggesting that AIB transmits aversive signals. Activated AIA interneurons may induce worms to cross the $\mathrm{Cu}^{2+}$ barrier to reach diacetyl by inhibiting AIB interneurons. Alternatively, AIA interneurons may release neuropeptides to modulate the neuronal circuit, as AWC sensory neurons release NLP-1 (Chalasani et al., 2010).

Interneurons play an important role in the processing of sensory information. In vertebrates, amacrine cells in the retina receive excitatory inputs from bipolar cells and mutually inhibit other bipolar cells (Cook and McReynolds, 1998). Furthermore, in Drosophila, interneurons receive many inputs from ORNs and make synapses onto the presynaptic terminals of ORNs, playing the role of gain control in the modulation of sensory sensitivity (Olsen and Wilson, 2008). These interneurons allow lateral interaction among signals sensed by distinct neurons, like AIA interneurons do in the sensory integration. Mammalian NPRs and their ligands are expressed in the layers of retina, including the amacrine cells (Blute et al., 2000; Abdelalim et al., 2008). The function of NPR-A and -B in these neurons could be analogous to that of GCY-28 in C. elegans.

In the future, if electrophysiological analyses can be applied in this system, we can understand more precise mechanisms of the sensory integration. Further analyses on the relationship between the neuronal properties of AIA interneurons and molecules that regulate the sensory integration may shed light on the fundamental neural mechanisms of sensory integration for behavioral choice in invertebrates and decision making in vertebrates.

\section{References}

Abdelalim EM, Masuda C, Tooyama I (2008) Expression of natriuretic peptide-activated guanylate cyclases by cholinergic and dopaminergic amacrine cells of the rat retina. Peptides 29:622-628.

Amet LE, Lauri SE, Hienola A, Croll SD, Lu Y, Levorse JM, Prabhakaran B, Taira T, Rauvala H, Vogt TF (2001) Enhanced hippocampal long-term potentiation in mice lacking heparin-binding growth-associated molecule. Mol Cell Neurosci 17:1014-1024.

Bargmann CI, Hartwieg E, Horvitz HR (1993) Odorant-selective genes and neurons mediate olfaction in C. elegans. Cell 74:515-527.

Baude EJ, Arora VK, Yu S, Garbers DL, Wedel BJ (1997) The cloning of a Caenorhabditis elegans guanylyl cyclase and the construction of a ligandsensitive mammalian/nematode chimeric receptor. J Biol Chem 272:16035-16039.

Bessereau JL, Wright A, Williams DC, Schuske K, Davis MW, Jorgensen EM (2001) Mobilization of a Drosophila transposon in the Caenorhabditis elegans germ line. Nature 413:70-74.

Bilsland JG, Wheeldon A, Mead A, Znamenskiy P, Almond S, Waters KA, Thakur M, Beaumont V, Bonnert TP, Heavens R, Whiting P, McAllister G, Munoz-Sanjuan I (2008) Behavioral and neurochemical alterations in mice deficient in anaplastic lymphoma kinase suggest therapeutic potential for psychiatric indications. Neuropsychopharmacology 33:685-700.

Blute TA, Lee HK, Huffmaster T, Haverkamp S, Eldred WD (2000) Localization of natriuretic peptides and their activation of particulate guanylate cyclase and nitric oxide synthase in the retina. J Comp Neurol 424:689-700.

Boulin T, Bessereau JL (2007) Mos1-mediated insertional mutagenesis in Caenorhabditis elegans. Nat Protoc 2:1276-1287.

Brenner S (1974) The genetics of Caenorhabditis elegans. Genetics 77:71-94.

Certel SJ, Savella MG, Schlegel DC, Kravitz EA (2007) Modulation of Drosophila male behavioral choice. Proc Natl Acad Sci U S A 104:4706-4711.

Chalasani SH, Chronis N, Tsunozaki M, Gray JM, Ramot D, Goodman MB, Bargmann CI (2007) Dissecting a circuit for olfactory behaviour in Caenorhabditis elegans. Nature 450:63-70.

Chalasani SH, Kato S, Albrecht DR, Nakagawa T, Abbott LF, Bargmann CI (2010) Neuropeptide feedback modifies odor-evoked dynamics in Caenorhabditis elegans olfactory neurons. Nat Neurosci 13:615-621.

Cho SW, Cho JH, Song HO, Park CS (2005) Identification and character- 
ization of a putative cyclic nucleotide-gated channel, CNG-1, in C. elegans. Mol Cells 19:149-154.

Chronis N, Zimmer M, Bargmann CI (2007) Microfluidics for in vivo imaging of neuronal and behavioral activity in Caenorhabditis elegans. Nat Methods 4:727-731.

Cook PB, McReynolds JS (1998) Lateral inhibition in the inner retina is important for spatial tuning of ganglion cells. Nat Neurosci 1:714-719.

Dwyer ND, Troemel ER, Sengupta P, Bargmann CI (1998) Odorant receptor localization to olfactory cilia is mediated by ODR-4, a novel membrane-associated protein. Cell 93:455-466.

Esposito G, Di Schiavi E, Bergamasco C, Bazzicalupo P (2007) Efficient and cell specific knock-down of gene function in targeted C. elegans neurons. Gene 395:170-176.

Gillette R, Huang RC, Hatcher N, Moroz LL (2000) Cost-benefit analysis potential in feeding behavior of a predatory snail by integration of hunger, taste, and pain. Proc Natl Acad Sci U S A 97:3585-3590.

Golden JW, Riddle DL (1984) The Caenorhabditis elegans dauer larva: developmental effects of pheromone, food, and temperature. Dev Biol 102:368-378.

Harbinder S, Tavernarakis N, Herndon LA, Kinnell M, Xu SQ, Fire A, Driscoll M (1997) Genetically targeted cell disruption in Caenorhabditis elegans. Proc Natl Acad Sci U S A 94:13128-13133.

Herring N, Paterson DJ (2009) Neuromodulators of peripheral cardiac sympatho-vagal balance. Exp Physiol 94:46-53.

Hilliard MA, Apicella AJ, Kerr R, Suzuki H, Bazzicalupo P, Schafer WR (2005) In vivo imaging of C. elegans ASH neurons: cellular response and adaptation to chemical repellents. EMBO J 24:63-72.

Inada H, Ito H, Satterlee J, Sengupta P, Matsumoto K, Mori I (2006) Identification of guanylyl cyclases that function in thermosensory neurons of Caenorhabditis elegans. Genetics 172:2239-2252.

Ishihara T, Iino Y, Mohri A, Mori I, Gengyo-Ando K, Mitani S, Katsura I (2002) HEN-1, a secretory protein with an LDL receptor motif, regulates sensory integration and learning in Caenorhabditis elegans. Cell 109:639-649.

Iwahara T, Fujimoto J, Wen D, Cupples R, Bucay N, Arakawa T, Mori S, Ratzkin B, Yamamoto T (1997) Molecular characterization of ALK, a receptor tyrosine kinase expressed specifically in the nervous system. Oncogene 14:439-449.

Kimura KD, Miyawaki A, Matsumoto K, Mori I (2004) The C. elegans thermosensory neuron AFD responds to warming. Curr Biol 14:1291-1295.

Kodama E, Kuhara A, Mohri-Shiomi A, Kimura KD, Okumura M, Tomioka M, Iino Y, Mori I (2006) Insulin-like signaling and the neural circuit for integrative behavior in C. elegans. Genes Dev 20:2955-2960.

Kristan WB (2008) Neuronal decision-making circuits. Curr Biol 18:R928-R932.

Lee HH, Norris A, Weiss JB, Frasch M (2003) Jelly belly protein activates the receptor tyrosine kinase Alk to specify visceral muscle pioneers. Nature 425:507-512.

Macosko EZ, Pokala N, Feinberg EH, Chalasani SH, Butcher RA, Clardy J, Bargmann CI (2009) A hub-and-spoke circuit drives pheromone attraction and social behaviour in C. elegans. Nature 458:1171-1175.

Mello C, Fire A (1995) DNA transformation. Methods Cell Biol 48:451-482.

Meredith MA (2002) On the neuronal basis for multisensory convergence: a brief overview. Brain Res Cogn Brain Res 14:31-40.

Miyawaki A, Llopis J, Heim R, McCaffery JM, Adams JA, Ikura M, Tsien RY (1997) Fluorescent indicators for $\mathrm{Ca}^{2+}$ based on green fluorescent proteins and calmodulin. Nature 388:882-887.

Mori I, Sasakura H, Kuhara A (2007) Worm thermotaxis: a model system for analyzing thermosensation and neural plasticity. Curr Opin Neurobiol 17:712-719.

Murphy GJ, Isaacson JS (2003) Presynaptic cyclic nucleotide-gated ion channels modulate neurotransmission in the mammalian olfactory bulb. Neuron 37:639-647.

Nagai T, Yamada S, Tominaga T, Ichikawa M, Miyawaki A (2004) Expanded dynamic range of fluorescent indicators for $\mathrm{Ca}^{2+}$ by circularly permuted yellow fluorescent proteins. Proc Natl Acad Sci USA 101:10554-10559.

Okochi Y, Kimura KD, Ohta A, Mori I (2005) Diverse regulation of sensory signaling by C. elegans nPKC-epsilon/eta TTX-4. EMBO J 24:2127-2137.
Olsen SR, Wilson RI (2008) Lateral presynaptic inhibition mediates gain control in an olfactory circuit. Nature 452:956-960.

Ortiz CO, Etchberger JF, Posy SL, Frøkjaer-Jensen C, Lockery S, Honig B, Hobert O (2006) Searching for neuronal left/right asymmetry: genomewide analysis of nematode receptor-type guanylyl cyclases. Genetics 173:131-149.

Ortiz CO, Faumont S, Takayama J, Ahmed HK, Goldsmith AD, Pocock R, McCormick KE, Kunimoto H, Iino Y, Lockery S, Hobert O (2009) Lateralized gustatory behavior of C. elegans is controlled by specific receptortype guanylyl cyclases. Curr Biol 19:996-1004.

Palmer RH, Vernersson E, Grabbe C, Hallberg B (2009) Anaplastic lymphoma kinase: signalling in development and disease. Biochem J 420:345-361.

Pavlov I, Rauvala H, Taira T (2006) Enhanced hippocampal GABAergic inhibition in mice overexpressing heparin-binding growth-associated molecule. Neuroscience 139:505-511.

Reiner DJ, Weinshenker D, Tian H, Thomas JH, Nishiwaki K, Miwa J, Gruninger T, Leboeuf B, Garcia LR (2006) Behavioral genetics of Caenorhabditis elegans unc-103-encoded erg-like $\mathrm{K}^{+}$channel. J Neurogenet 20:41-66.

Reiner DJ, Ailion M, Thomas JH, Meyer BJ (2008) C. elegans anaplastic lymphoma kinase ortholog SCD-2 controls dauer formation by modulating TGF-beta signaling. Curr Biol 18:1101-1109.

Sambongi Y, Nagae T, Liu Y, Yoshimizu T, Takeda K, Wada Y, Futai M (1999) Sensing of cadmium and copper ions by externally exposed ADL, ASE, and ASH neurons elicits avoidance response in Caenorhabditis elegans. Neuroreport 10:753-757.

Schall JD (2001) Neural basis of deciding, choosing and acting. Nat Rev Neurosci 2:33-42.

Sengupta P, Chou JH, Bargmann CI (1996) odr-10 encodes a seven transmembrane domain olfactory receptor required for responses to the odorant diacetyl. Cell 84:899-909.

Shaw BK, Kristan WB Jr (1997) The neuronal basis of the behavioral choice between swimming and shortening in the leech: control is not selectively exercised at higher circuit levels. J Neurosci 17:786-795.

Sieburth D, Ch'ng Q, Dybbs M, Tavazoie M, Kennedy S, Wang D, Dupuy D, Rual JF, Hill DE, Vidal M, Ruvkun G, Kaplan JM (2005) Systematic analysis of genes required for synapse structure and function. Nature 436:510-517.

Sieburth D, Madison JM, Kaplan JM (2007) PKC-1 regulates secretion of neuropeptides. Nat Neurosci 10:49-57.

Suzuki H, Kerr R, Bianchi L, Frøkjaer-Jensen C, Slone D, Xue J, Gerstbrein B, Driscoll M, Schafer WR (2003) In vivo imaging of C. elegans mechanosensory neurons demonstrates a specific role for the MEC-4 channel in the process of gentle touch sensation. Neuron 39:1005-1017.

Suzuki H, Thiele TR, Faumont S, Ezcurra M, Lockery SR, Schafer WR (2008) Functional asymmetry in Caenorhabditis elegans taste neurons and its computational role in chemotaxis. Nature 454:114-117.

Svenssona GP, Löfstedt C, Skals N (2007) Listening in pheromone plumes: disruption of olfactory-guided mate attraction in a moth by a bat-like ultrasound. J Insect Sci 7:59.

Tomioka M, Adachi T, Suzuki H, Kunitomo H, Schafer WR, Iino Y (2006) The insulin/PI 3-kinase pathway regulates salt chemotaxis learning in Caenorhabditis elegans. Neuron 51:613-625.

Tsunozaki M, Chalasani SH, Bargmann CI (2008) A behavioral switch: cGMP and PKC signaling in olfactory neurons reverses odor preference in C. elegans. Neuron 59:959-971.

Wakabayashi T, Kitagawa I, Shingai R (2004) Neurons regulating the duration of forward locomotion in Caenorhabditis elegans. Neurosci Res 50:103-111.

White JG, Southgate E, Thomson JN, Brenner S (1986) The structure of the nervous system of the nematode Caenorhabditis elegans. Philos Trans R Soc Lond B Biol Sci 314:1-340.

Wicks SR, de Vries CJ, van Luenen HG, Plasterk RH (2000) CHE-3, a cytosolic dynein heavy chain, is required for sensory cilia structure and function in Caenorhabditis elegans. Dev Biol 221:295-307.

Zimmer M, Gray JM, Pokala N, Chang AJ, Karow DS, Marletta MA, Hudson ML, Morton DB, Chronis N, Bargmann CI (2009) Neurons detect increases and decreases in oxygen levels using distinct guanylate cyclases. Neuron 61:865-879. 